\title{
Outcomes of 4 years of molecular genetic diagnosis on a panel of genes involved in premature aging syndromes, including laminopathies and related disorders
}

Maude Grelet ${ }^{1,2}$, Véronique Blanck ${ }^{1}$, Sabine Sigaudy ${ }^{1,2}$, Nicole Philip ${ }^{1,2}$, Fabienne Giuliano ${ }^{3}$, Khaoula Khachnaoui ${ }^{3}$, Godelieve Morel ${ }^{4,5}$, Sarah Grotto ${ }^{6}$, Julia Sophie ${ }^{7}$, Céline Poirsier ${ }^{8}$, James Lespinasse ${ }^{9}$, Laurent Alric ${ }^{10}$, Patrick Calvas ${ }^{7}$, Gihane Chalhoub ${ }^{11}$, Valérie Layet ${ }^{12}$, Arnaud Molin ${ }^{13}$, Cindy Colson ${ }^{13}$, Luisa Marsili ${ }^{14}$, Patrick Edery ${ }^{4,5}$, Nicolas Lévy ${ }^{1,2,15}$ and Annachiara De Sandre-Giovannoli ${ }^{1,2,15^{*}}$ (D)

\begin{abstract}
Background: Segmental progeroid syndromes are a heterogeneous group of rare and often severe genetic disorders that have been studied since the twentieth century. These progeroid syndromes are defined as segmental because only some of the features observed during natural aging are accelerated.

Methods: Since 2015, the Molecular Genetics Laboratory in Marseille La Timone Hospital proposes molecular diagnosis of premature aging syndromes including laminopathies and related disorders upon NGS sequencing of a panel of 82 genes involved in these syndromes.

We analyzed the results obtained in 4 years on 66 patients issued from France and abroad.

Results: Globally, pathogenic or likely pathogenic variants (ACMG class 5 or 4) were identified in about 1/4 of the cases; among these, 9 pathogenic variants were novel. On the other hand, the diagnostic yield of our panel was over $60 \%$ when the patients were addressed upon a nosologically specific clinical suspicion, excepted for connective tissue disorders, for which clinical diagnosis may be more challenging. Prenatal testing was proposed to 3 families. We additionally detected 16 variants of uncertain significance and reclassified 3 of them as benign upon segregation analysis in first degree relatives.

Conclusions: High throughput sequencing using the Laminopathies/ Premature Aging disorders panel allowed molecular diagnosis of rare disorders associated with premature aging features and genetic counseling for families, representing an interesting first-level analysis before whole genome sequencing may be proposed, as a future second step, by the National high throughput sequencing platforms ("Medicine France Genomics 2025" Plan), in families without molecular diagnosis.
\end{abstract}

Keywords: Progeroid, Panel, Pathogenic,variant

\footnotetext{
* Correspondence: annachiara.desandre@ap-hm.fr

${ }^{1}$ Department of Medical Genetics, Assistance Publique Hopitaux de Marseille,

Marseille, France

${ }^{2}$ Aix Marseille Univ, INSERM, MMG, Marseille, France

Full list of author information is available at the end of the article
}

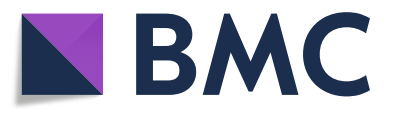

(c) The Author(s). 2019 Open Access This article is distributed under the terms of the Creative Commons Attribution 4.0 International License (http://creativecommons.org/licenses/by/4.0/), which permits unrestricted use, distribution, and reproduction in any medium, provided you give appropriate credit to the original author(s) and the source, provide a link to the Creative Commons license, and indicate if changes were made. The Creative Commons Public Domain Dedication waiver (http://creativecommons.org/publicdomain/zero/1.0/) applies to the data made available in this article, unless otherwise stated. 


\section{Background}

Segmental progeroid syndromes are a heterogeneous group of rare genetic disorders, which were clinically described in medical journals since the twentieth century. These syndromes are defined as segmental because only some of the aging features are accelerated [1]. Historically, and based on their main pathophysiological bases, three major groups of progeroid syndromes may be distinguished.

The first consists of syndromes due to alteration of DNA-repair genes. The RecQ family genes, encoding DNA helicases, are mainly involved in this group of syndromes [2, 3]. DNA helicases are enzymes involved in different DNA-repair processes and are referred to as guardians of the genome. They play important roles in genome stability and maintenance [4]. Several syndromes are associated to the RecQ genes' family including Werner syndrome (WS), Bloom syndrome (BS) and Rothmund Thomson syndrome (RTS) [5-7], that are respectively associated with pathogenic variants in RECQL2/WRN, RECQL3/BLM, and RECQL4 genes. These syndromes share autosomal recessive inheritance and an increased susceptibility to cancer. WS is an adult-onset progeria syndrome, whereas the first symptoms of BS and RTS appear in childhood. Cockayne syndrome is another autosomal recessive disorder caused by mutations of DNA-repair genes, the CS proteins. Pathogenic variants of CSA/ERCC8 and CSB/ERCC6 genes are responsible for the majority of cases $[1,8,9]$.

The second group deals with proteins maintaining the integrity of the nuclear envelope. Lamins $\mathrm{A} / \mathrm{C}$ and their protein partners are mainly involved in this group of disorders. In 2003, De Sandre-Giovannoli et al. and Eriksson et al. identified a synonymous point mutation of the LMNA gene (NM_170707.4: c.1824C > T; p.Gly608Gly) as causative of the disease in patients affected with Hutchinson Gilford Progeria Syndrome (HGPS) [10, 11]. This synonymous and apparently harmless heterozygous variant activates a cryptic donor splice site in LMNA pre-mRNAs, leading to the production of a prelamin A precursor that lacks the cleavage recognition site for the endoprotease ZMPSTE24. As a result, a truncated and permanently prenylated prelamin A isoform named progerin accumulates and exerts multiple toxic effects in cells' nuclei [10-12]. Other LMNA mutations affecting prelamin A maturation result in more or less severe progeroid syndromes, called HGPS-like, depending essentially on the quantities of progerin/prelamin A isoforms produced [13]. Two other syndromes, restrictive dermopathy (RD), a perinatal lethal genodermatosis, and type $\mathrm{B}$ mandibuloacral dysplasia (MAD-B), a relatively milder progeroid syndrome characterized by skeletal, metabolic and cutaneous abnormalities and lipodystrophy, have also been associated to pathological accumulation of prelamin A. Recessive pathogenic variants in ZMPSTE24 have mostly been described in these syndromes, broadening the spectrum of prelamin- $\mathrm{A}$ associated disorders [1, 14-19]. Furthermore, several atypical progeroid syndromes (APS) or atypical Werner syndrome (AWS) with clinical features overlapping with HGPS and other prelamin A-linked disorders have been associated to missense mutations in the LMNA gene, which are often private and are seen in only a single or few families [20-27].

The third group is composed of syndromes characterized by features of premature aging resulting from diverse pathophysiological processes. This group includes all the disorders listed in Additional file 1: Table S1 and not belonging to the 1st and 2nd major groups of progeroid syndromes. It also includes Ehlers Danlos syndromes (EDS), a clinically and genetically heterogeneous group of diseases that affect connective tissues. EDS are classified into 13 subtypes according to the inheritance pattern, phenotype and pathogenetic mechanisms [28] and some of its subtypes present with premature aging signs. Cutis Laxa $(\mathrm{CL})$ syndromes presenting as well with progeroid features are also included in this third group [29].

Since 2015, the molecular genetics laboratory in Marseille La Timone Hospital proposes molecular diagnosis of premature aging syndromes as well as laminopathies and related syndromes upon NGS (next generation sequencing) analysis of a panel of 82 genes involved in those disorders (Additional file 1: Table S1). To the best of our knowledge, this panel is unique in France (Fig. 1) and in Europe (https://www.orpha.net/consor/cgi-bin/ClinicalLabs. php?lng=FR).

We report herein the outcomes of 4 years of NGS molecular diagnosis in 66 index cases coming from France and abroad, affected with syndromes featuring premature aging, and attempt to provide a critical discussion of the results obtained.

\section{Materials and methods}

Next generation sequencing and mutation identification procedures

In a diagnostic setting, we performed the targeted analysis of 82 genes (Additional file 1: Table S1). The genes were included into the panel because of their association with laminopathies or with diseases including premature ageing features; they were chosen upon bibliographic search (PubMed) and using genetic/clinical databases (namely OMIM and Orphanet).

Since 2015, 66 index cases were subjected to the NGS analysis and included in this study. The criteria used to include patients in the molecular study were one of the following: (i) patients whose clinical diagnosis fitted with one of the disorders associated with a gene of the panel (Additional file 1: Table S1), whether it was a progeroid syndrome or not, but having a specific nosologic 


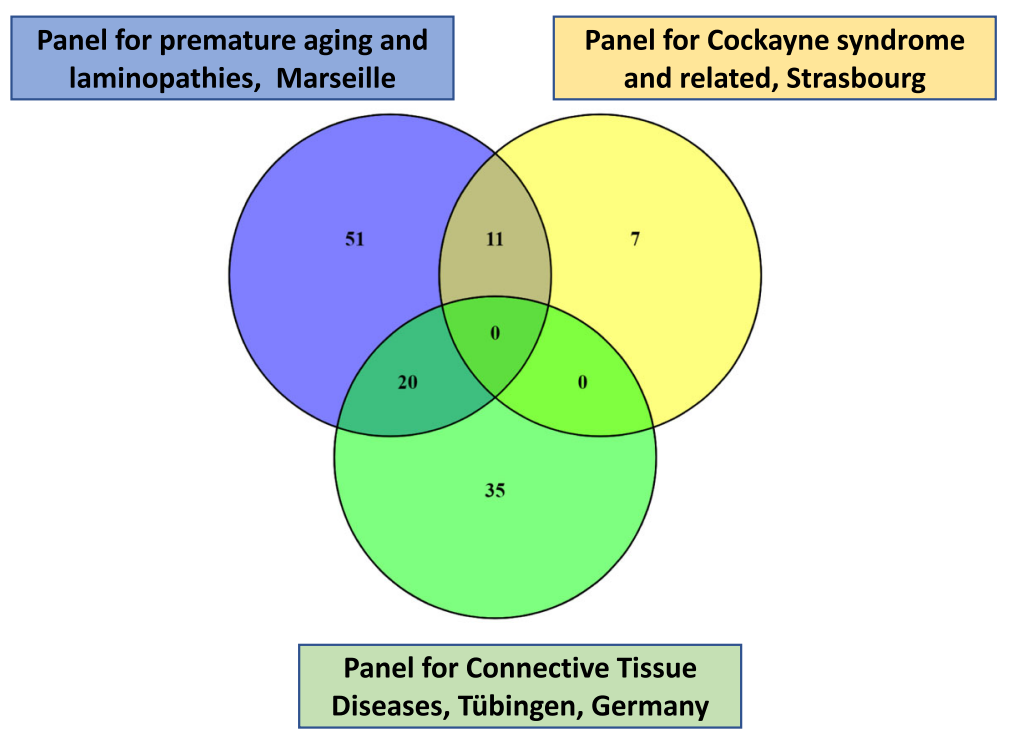

Fig. 1 Venny diagrams showing the existing overlaps among available gene lists in a diagnostic setting according to Orphanet, available to test patients with premature aging disorders (source: https://www.orpha.net/consor/cgi-bin/index.php) and data available on the laboratories' websites (https://www.cegat.de/en/diagnostics/diagnostic-panels/connective-tissue-diseases/; http://www.chru-strasbourg.fr/sites/default/files/u1 1 O/NER_18genes_190308_2.pdf). We compare our gene panel with a panel of 55 genes asssociated with connective tissue diseases in Tübingen, Germany (CeGaT GmbH) and a panel of 18 genes associated with Cockayne and related syndromes, in Strasbourg, France (CHU de Strasbourg, Hôpital Civil)

classification, (ii) patients for whom the prescribing physician suspected a disease related to premature aging, due the association of at least two premature aging signs, but without a specific nosologic classification.

DNA was extracted from peripheral blood samples by the Biological Resources Center CRB-TAC (with NF-S 86-900 and ISO-9001 V2015 certifications) of the Department of Medical Genetics.

We created a SureSelectXT $12-24 \mathrm{Mb}$ library, custom 1032 gene panel in collaboration with Agilent Technologies (Santa Clara, California, USA), to be used for enrichment of targeted sequences for several molecular diagnosis indications in the laboratory. The coding regions and flanking intronic regions of the 1032 genes were enriched, in solution, using the SureSelect Target Enrichment System from Agilent (Santa Clara, California, USA), following the manufacturer recommendations. The Ion Proton platform (Thermo Fisher Scientific, USA) was used for high throughput sequencing. Then, raw data were converted to Fastq files and aligned to the reference sequence of the human genome (University of California Santa Cruz, hg19/GRCh37, (https:// genome.ucsc.edu/), using the Torrent Suite software (Thermo Fisher). The same software was used to perform variant calling (germline_low_stringency_targetseq), with the following parameters (min_cov_each_strand: 0 , min variant_score: 10, min_allele_freq: 0.1, snp_min_coverage: 6 snp and indel; strand_bias: $0.98 \mathrm{snp}$ and 0.85 indel). A variant calling format (VCF) file and binary alignment map and index (BAM/BAI) files were then obtained and used for variant annotation using the in-house software VarAFT (Variant Annotation and Filtration Tool, https://varaft.eu/) [30]). The list of genes to be analyzed for each patient was also selected using dedicated bedfiles by VarAFT. Sequence reads and variants' visualization was performed using IGV (https://software.broadinstitute.org/software/igv/) [31].

The BEDTools-based VarAFT coverage module was used to compute breadth and depth of coverage data using BAM files for the selected gene list, producing a coverage report. Nucleotidic positions were interpreted only over a cutoff of 20X reads depth.

Variants whose allele frequencies were $>1 \%$ in GnomAD (https://gnomad.broadinstitute.org) and 1000 genomes (http://www.internationalgenome.org/) were removed, as well as deep intronic variants. The remaining variants were analyzed according to their genomic position and their predicted effects on RNAs and proteins. In order to study the pathogenicity of the variants, we used population databases such as gnomAD (https://gnomad.broadinstitute.org/), sequence databases such as ClinVar (https://www.ncbi.nlm. nih.gov/clinvar/), LOVD (http://www.lovd.nl/3.0/home), disease databases such as OMIM (https://www.omim.org/), integrated databases as ClinGen and in silico predictive algorithms such as Mutation Taster (http://www.mutationtaster. org/), Human Splicing Finder (http://www.umd.be/HSF3/) and UMD Predictor (http://umd-predictor.eu/), together with a review of the literature [32-34].

The variants were classified as "pathogenic", "likely pathogenic", "of uncertain significance", "likely benign" 
or "benign" (respectively classes 5 to 1 ) according to the American College of Medical Genetics and Genomics (ACMG) classification, using Intervar (http://wintervar. wglab.org/), ClinVar (https://www.ncbi.nlm.nih.gov/clinvar/) and ClinGen (https://www.clinicalgenome.org/); the classification was manually adjusted using further ACMG criteria, whenever necessary, depending on the familial context and literature data [35]. In each case, the additional ACMG criteria allowing the reclassification of variants are given in Table 1 .

Only the variants of classes 5 to 3 were reported in molecular genetics diagnostic reports after Sanger sequencing confirmation. Genes were named following the Hugo gene nomenclature committee guidelines (https://www.genenames.org/); DNA mutations and predicted protein changes were named following the HGVS nomenclature guidelines (http://www.hgvs. org/mutnomen/).

\section{Sanger sequencing for the validation of variants}

We designed primers for PCR amplification using the Primer3 software (http://bioinfo.ut.ee/primer3/) in order to amplify the gene's exon. Briefly, Sanger sequencing was performed as follows: purification of PCR products was performed according to the manufacturer's instructions and both strands were sequenced using the Big Dye Terminator V.1.1 Cycle Sequencing Kit (Applied Biosystems). Sequence reactions were purified on Sephadex G50 (Amersham Pharmacia Biotech, Foster City, California, USA). We performed capillary electrophoresis on a Genetic Analyzer ABI3500XL (Life Technologies, USA). Electropherograms were analyzed on the Sequence Analysis Software V.5.2 (Applied Biosystems) and aligned and interpreted relative to the reference sequence using Sequencher V.5.4.6 (Gene Codes Corporation).

\section{Results}

Since 2015, we analyzed 66 patients from all over France and abroad on a panel of genes involved in premature aging syndromes. As depicted in Fig. 1, 50\% of the patients were addressed from the southern regions of France and only 3\% from abroad (Fig. 2). To the best of our knowledge, this kind of gene panel largely exploring premature aging syndromes and laminopathies is unique in France and Europe (https://www.orpha.net/consor/ cgi-bin/index.php). Some overlap exists with other gene panels, specifically exploring sets of genes involved in particular diseases such as for example connective tissue disorders or Cockayne and related syndromes (Fig. 1).

The average coverage obtained at $20 \mathrm{X}$ on the list of genes and corresponding reference transcripts we describe (Additional file 1: Table S1) was 93\% $(248,228 / 267,610$ nucleotides covered at $20 \mathrm{X}+/-10,237$ mean nucleotides, i.e. $+/-4 \%$ mean $\mathrm{SD}$ ).
The molecular analysis allowed to identify 20 pathogenic or likely pathogenic variants in 16 patients according to ACMG, using Intervar (http://wintervar.wglab.org/), literature and databases, as described above (Table 1). Whenever necessary, the clinical interpretation of sequence variants was adjusted manually according to the literature, genetic databases and patients' clinical data.

Globally, class 1-2 (benign and likely benign) variants were identified in $59 \%$ of the patients, class 3 variants (VUS, variant of unknown significance) in $15 \%$ and class 5 and 4 variants (pathogenic or likely pathogenic, respectively) in $26 \%$ of the patients (about $1 / 4$ ). In order to analyze the molecular diagnostic yield with respect to the nosologic specificity of the clinical suspicion, we divided the index cases in two, then in three groups according to the clinical diagnosis/indication for NGS: respectively "specific clinical suspicion and unspecified progeroid disorders" then "specific clinical suspicion other than connective tissue disorders, connective tissue disorders (namely including CL and EDS) and unspecified progeroid disorders" (Fig. 3a, b). As expected, when considering the first group of patients with "specific clinical suspicion" we obtained a 39\% diagnostic yield for ACMG classes 4 and 5 variants (likely pathogenic or pathogenic), compared to the "unspecified progeroid disorders" for which the same types of variants were retrieved only in $10 \%$ of the patients. When we further divided the first group in two, i.e. "specific clinical suspicion other than connective tissue disorders and connective tissue disorders", we obtained a much higher diagnostic yield (62\%) with ACMG classes 4 and 5 variants for patients with a nosologically defined clinical syndrome vs only $26 \%$ for connective tissue disorders (Fig. $3 b$ ). In the same graph, we can see that the detection rate of class 3 variants was the same for the two subgroups.

Table 1 compiles the 36 variants observed in our cohort from class 3 to 5 ; 9 of the 20 variants belonging to classes 4 and 5 are described in this report for the first time (Table 1, last column "THIS REPORT").

In the group of nosologically defined, suspected clinical syndromes, we identified compound heterozygous pathogenic variants of RecQ family genes for three patients: RECQL4 in two patients (P2 and P6) presenting with RTS and WRN/RECQL2 in one patient with WS (P8). Both patients with RTS were female and had intrauterine and postnatal growth retardation, poikiloderma (collectively defining the following skin anomalies: reticulated hypo- and hyperpigmentation, punctate atrophy and telangiectasias) (Fig. 4B1-B3) and skeletal anomalies (fractures for patient P2 and decreased bone mineral density for patient P6). Patient P2 was older than patient P6, she developed a metastatic colon cancer and died at age 27. The pathogenic heterozygous variants found in patient P6 had already been described in a 


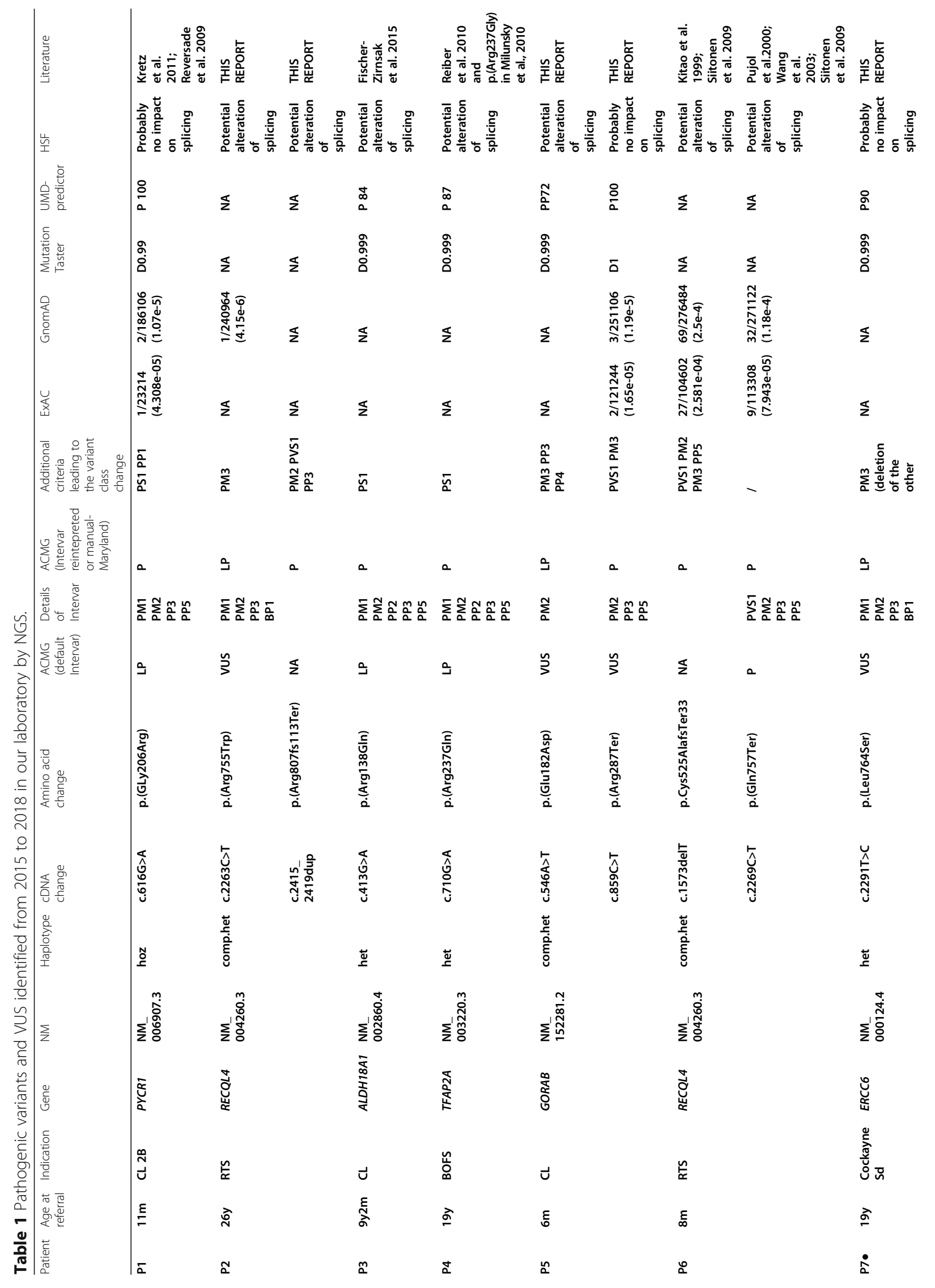




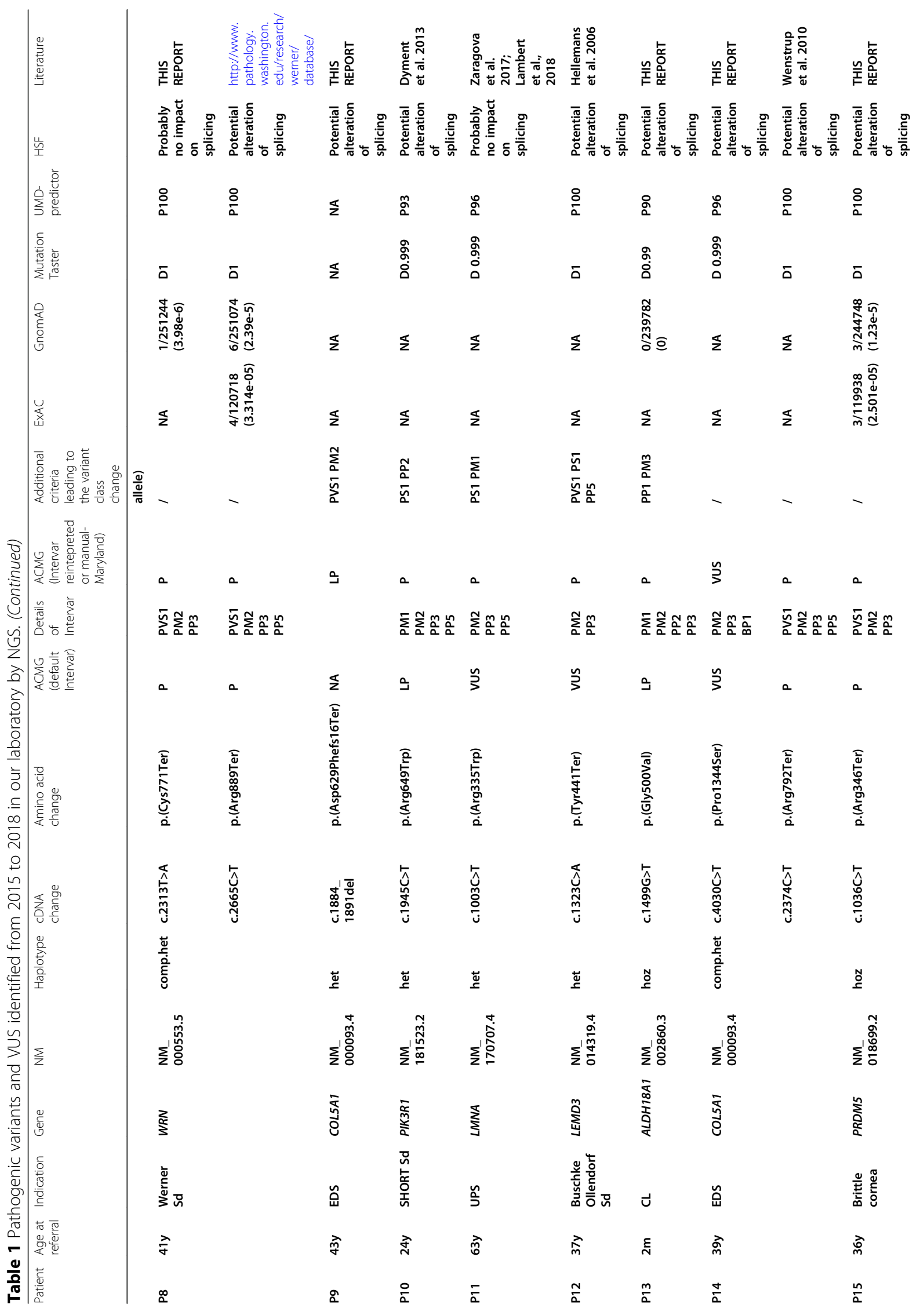




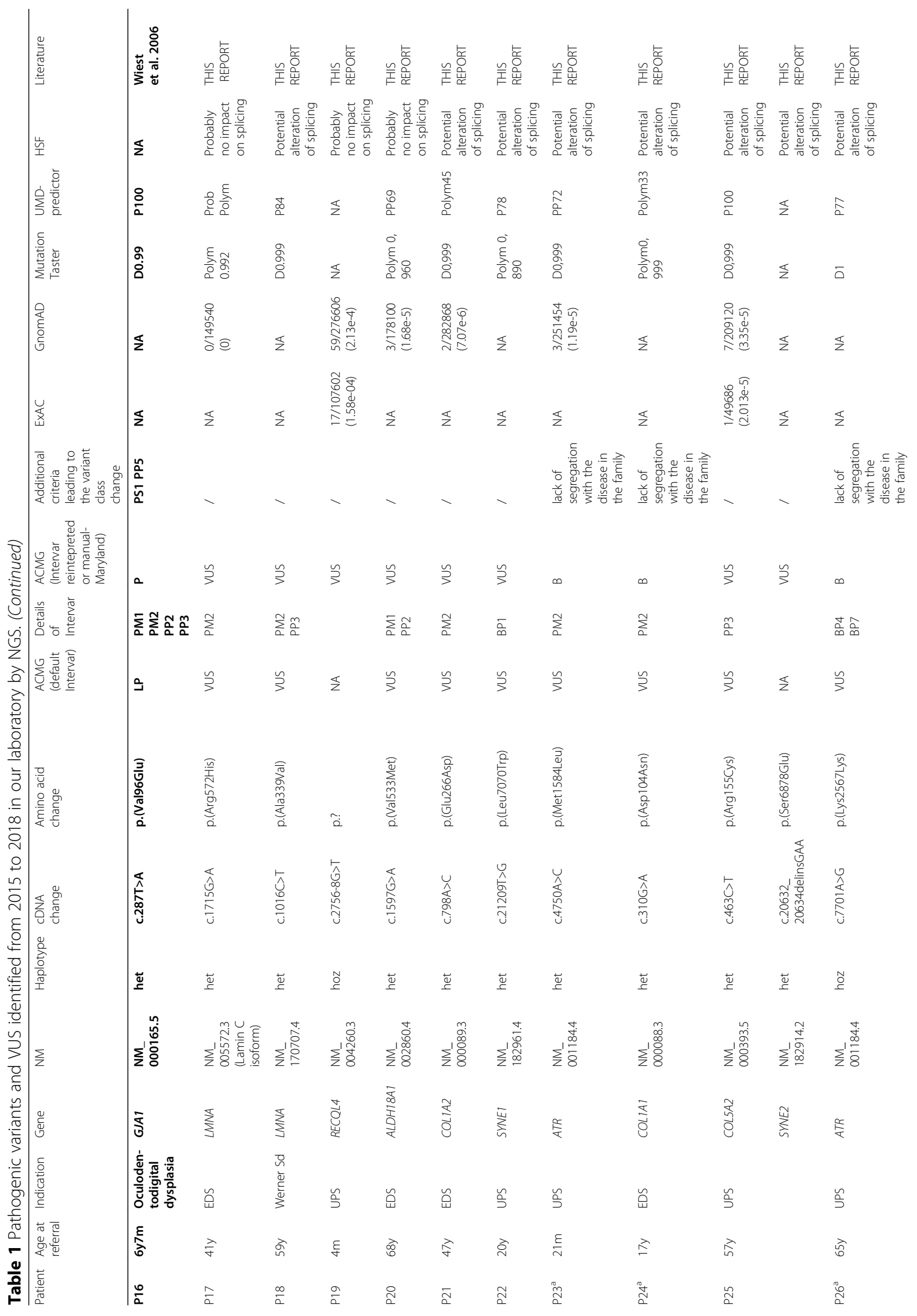




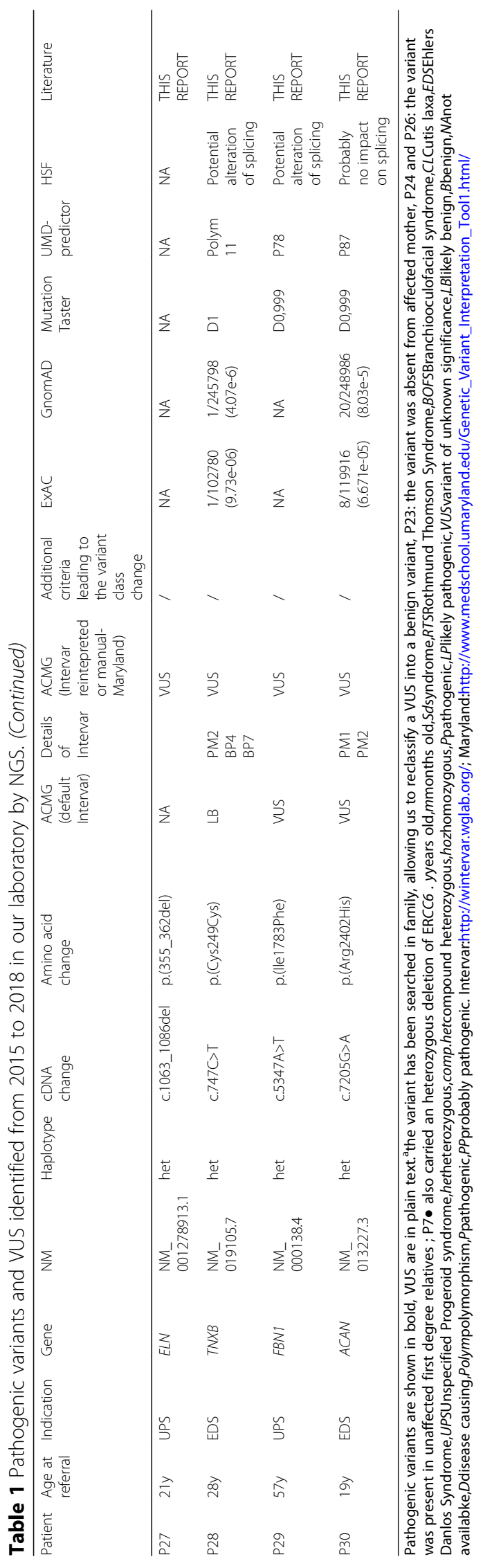



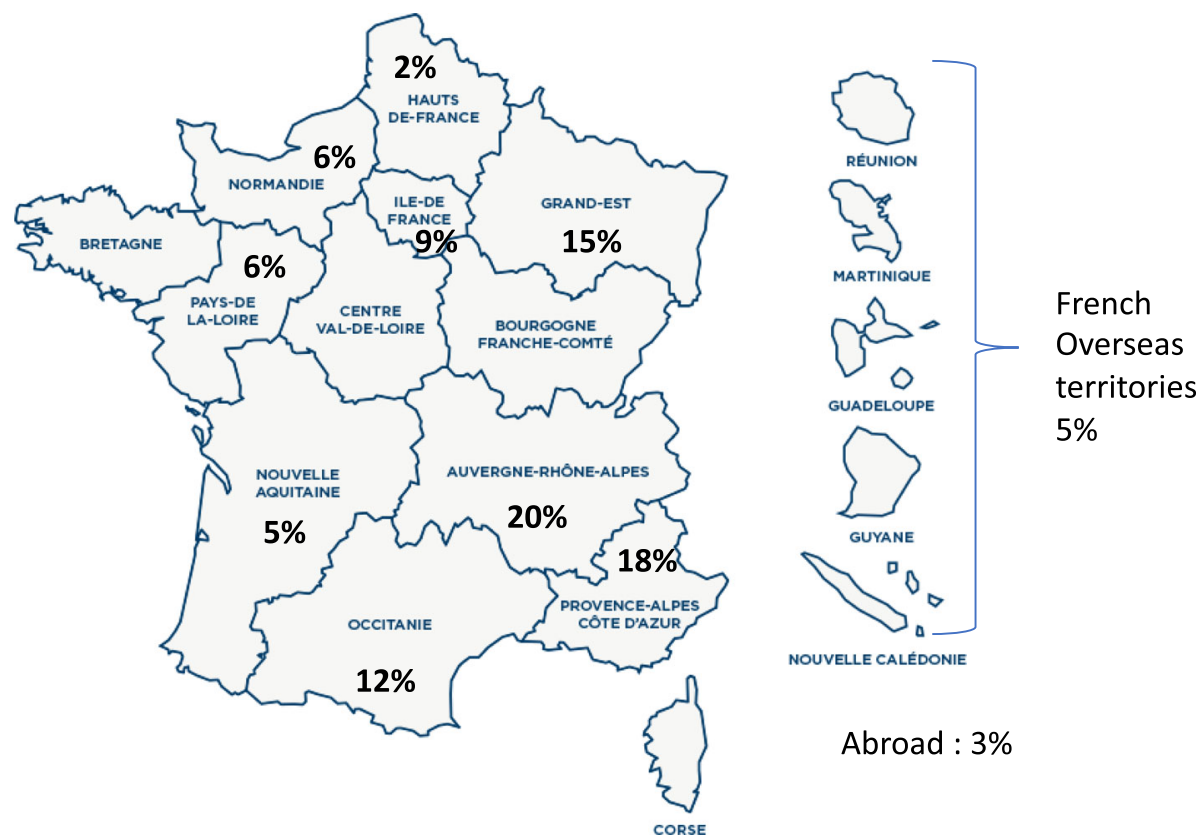

Abroad : $3 \%$

Fig. 2 Map of France depicting the origin of index cases

patient presenting with RTS [36-38], whereas the two pathogenic variants observed in patient P2 (c.2263C > T; p.(Arg755Trp) and c.2415_2419dup; p.(Arg807fs113Ter)) are described for the first time in this report. Patient P8 affected with WS presented with bilateral cataract, premature graying of scalp hair, alterations of the skin (thin skin and hyperpigmentation), soft tissue calcification, Achille's tendinopathy, muscle atrophy and hypothyroidism at age 38 . One of the pathogenic variants identified in the WRN gene (c.2313 T > A; p.(Cys771Ter)) is novel as well. Another patient (P7) was referred to our laboratory with clinical features of late onset Cockayne Syndrome. A deletion of 4,8 Mb encompassing the ERCC6 gene was already identified by array CGH and we observed a missense variant in exon 12 on the other ERCC6 allele (c.2291 T > C; p.(Leu764Ser)).

We identified already described pathogenic variants confirming the clinical diagnoses of SHORT Syndrome in patient $\mathrm{P} 10$, of Buschke-Ollendorf syndrome in patient P12, of Branchio-Oculo-Facial Syndrome (BOFS) in patient $\mathrm{P} 4$ and of Oculodentodigital Dysplasia in patient P16 (Table 1), respectively in the PIK3R1, LEMD3/

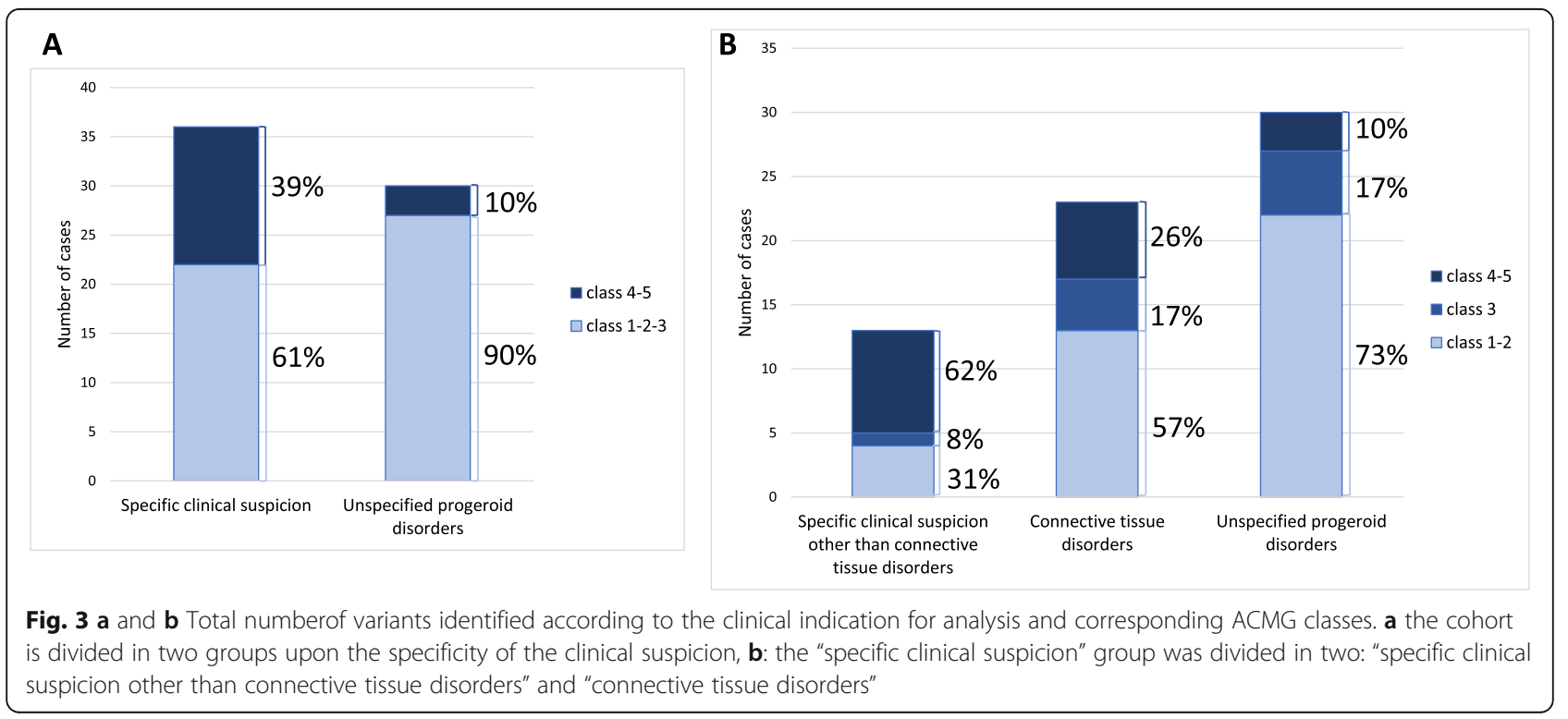




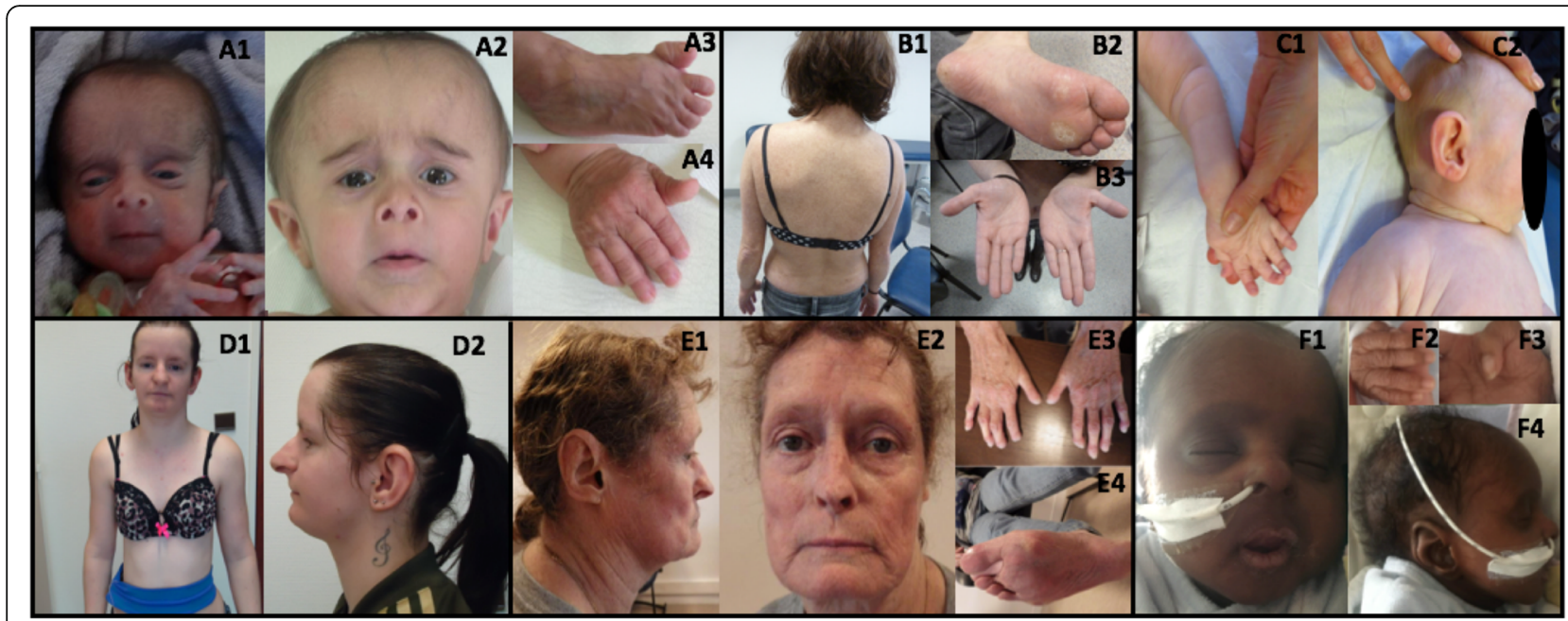

Fig. 4 Pictures of patients P1, P2, P5, P10, P11, P13. A1-A4: Patient P1, on A1 ten days after birth and on A2-A4 at the age of 11 months, affected with De Barsy syndrome (PYCR1: homozygous C.616G>A); B1-B3 : Patient P2 affected with Rothmund-Thomson syndrome at the age of 26 (RECQL4: c.2263C>T and c.2415_2419dup); C1, C2: Patient P5 affected with Geroderma Osteodysplastica at the age of 5 months (GORAB: C.546A>T and C.859C>T); D1, D2: Patient P10 affected with SHORT syndrome (PIK3R1: C.1945C>T) at the age of 23 ; E1-E4 : Patient P11 at the age of 63, affected with a progeroid laminopathy (LMNA: C.1003C>T); F1-F4 : Patient P13 affected with cutis laxa (ALDH18A1: homozygous C.1499G>T) at the age of 1 month

$M A N 1, T F A P 2 A$ and GJA1 genes [39-42]. As it can be seen in Fig. 4D1, D2, patient P10 affected with SHORT syndrome presented with short stature (below the 3rd SD), triangular face with prominent forehead, deep-set eyes, narrow tip of the nose, low-hanging columella and partial lipodystrophy at age 23. She also presented Axenfeld-Rieger anomaly, diabetes mellitus, hearing loss and ovarian cysts.

In the group of connective tissue disorders, we identified new compound heterozygous or homozygous class 4 or 5 variants in patients presenting with Cutis Laxa: GORAB (c.546A > T; p.(Glu182Asp)) and c.859C > T; p.(Arg287Ter)) for patient P5 and an homozygous novel variant in ALDH18A1 (c.1499G > T; p.(Gly500Val)) for patient P13 (Table 1 and Fig. 4F1-F4). Patient P5 was evaluated in his first year of life: he showed joint laxity, bilateral congenital hip dislocation, fracture of the tibia, skin wrinkling, premature aged appearance of the face, deep set eyes, droopy cheeks and a pinched nose (Fig. 4C1, C2). His nonsense variant was inherited from the mother, who presented with a mild phenotype associating joint laxity and a pinched nose. GORAB compound heterozygous variants and the clinical features of patient P5 were compatible with Geroderma osteodyplastica (GO) or Autosomic Recessive Cutis Laxa type 2 (ARCL2) diagnoses. Patient P13 was the first child of a consanguineous couple from Senegal; IUGR (intrauterine growth retardation) was observed during pregnancy. He was born prematurely with height and weight below the third centile and his head circumference was between the 5th and 10th percentile. Clinical examination at 1 month of life evidenced retarded postnatal growth (below the 3rd centile, as at birth), hypotonia, failure to thrive, large fontanelles, facial progeroid appearance and cutis laxa. He also presented stenosis of the aortic arch. ALDH18A1 pathogenic variants are responsible of De Barsy syndrome, previously known as ARCL3A, defining a molecular diagnosis that was compatible with the clinical features of patient P13 [29]. Other pathogenic variants already described in the literature confirmed the diagnosis of CL for patients P1 and P3 (Table 1 and Fig. 4A1-A4 for patient P1) [43-45]. Patient P1 (Fig. 4A1-A4) presented with aged appearance, a prominent forehead, loss of adipose tissue, translucent and wrinkly skin with visible veins. He also showed hypotonia and prenatal and postnatal growth retardation with conserved head circumference. This variant had already been associated with autosomal recessive Cutis Laxa [43, 44]. As mentioned above, EDS is another clinically and genetically heterogeneous group of connective tissue disorders. We identified two heterozygous nonsense variants in COL5A1 in two patients presenting with classical EDS: one of them (c.1884_1891del; p.(Asp629Phefs16Ter) is described in this report for the first time, in patient P9, while the other (c.2374C > T; p.(Arg792Ter) [46]) was observed in a compound heterozygous state with a novel missense VUS in the same gene: (c.4030C > T; p.(Pro1344Ser)) in patient P14 (Table 1). Brittle Cornea syndrome is classified as one of the EDS subtypes [28, 42,47]. In our cohort, patient P15, carried a homozygous nonsense variant in PRMD5 that was not reported before (c.1036C > T; p.(Arg346Ter), Table 1). Patient P15 was issued from a consanguineous union; at the age of 35 years, he presented 
with bilateral keratoconus, keratoglobus with bilateral corneal transplants, bilateral cataract surgery, blue sclerae, hearing loss, scoliosis and joint laxity.

We additionally observed $3 L M N A$ variants, of which one was considered as pathogenic based on previous publications (c.1003C > T; p.(Arg335Trp)) [48-54]. This variant was previously described in two patients presenting with acro-osteolysis and dilated cardiomyopathy (DCM), another with a cardiac phenotype, acro-osteolysis and hypertriglyceridemia and other patients presenting with isolated DCM [48-52]. As described in Lambert et al., [54] patient P11 presented with a severe progeroid phenotype including acro-osteolysis with painful articulations of hands and feet, she had a pace-maker for heart rhythm disturbances (atrial fibrillation and atrioventricular block) without DCM on echocardiograms and she reported that children laughed at her at school for her "aged face and skin" [54]. Patient P11, shown in Fig. 4E1-E4 at 63 years old, presented with a pinched nose, malar hypoplasia, and an emaciated aspect of the face with aged, thin and dyspigmented skin, brachydactyly due to osteolysis predominating on the first three distal phalanges of both hands, and xerosis of the skin.

We additionally identified 16 variants of unknown significance (VUS, ACMG class 3 variants). Among them, 3 were reclassified as benign upon segregation analysis on DNAs of first-degree relatives. In the remaining cases, we didn't receive parental DNAs to help reclassify them. Almost all VUS were identified in patients referred to our laboratory for Ehlers Danlos syndrome or unspecified progeroid syndromes. Among the suspected EDS, at least two patients (P20 and P28) had hypermobile EDS for which no gene was identified yet [28].

As mentioned above, one VUS in COL5A1 was associated with a pathogenic variant of the same gene in patient P14. The patient almost fulfilled the diagnostic criteria of Classical-Like EDS (clEDS) syndrome, i.e. as major criteria: skin hyperextensibility, general joint hypermobility (Beighton score: 8/9), spontaneous ecchymoses, autosomal recessive inheritance because she was born form a consanguineous couple and minor criteria: hand acrogeria with clinodactyly, pes planus, hallux valgus. However, she also presented atrophic scarring which is a major criterion for Classical EDS (cEDS) and is normally absent from clEDS. She had one brother with clinical signs of EDS. We did not identify pathogenic variants in TNXB (whose coding sequence NM 019105.6 was entirely covered by the genomic sequencing), but we observed one COL5A1 already known pathogenic variant (c.2374C > T; p.(Arg792Ter)) and one novel VUS (c.4030C > T; p.(Pro1344Ser)) [46]. Compound heterozygosity of COLSA1 has already been described in one family, with one missense COL5A1 variant playing the role of a "modifier gene" $[55,56]$. In order to determine the impact of the VUS, the analysis of its segregation in the patient's family would be useful.

Finally, two unreported heterozygous VUS that we would like to report were identified in the LMNA gene. One of these VUS (c.1016C > T; p.(Ala339Val)) was identified in patient P18, a 59 years old man, who displayed a range of premature-aging symptoms: premature balding since his twenties, short stature (-2.5 SD), low body weight $(-2.25$ DS), atrophic skin, sparse eyebrows and eyelashes, prominent eyes, micrognathia, and sloping shoulders. He suffered from intracranial hemorrhage at age 47 due to a ruptured posterior inferior cerebellar artery aneurysm. He also presented with aortic valve calcification associated with mild aortic insufficiency, incomplete right bundle branch block, hypertension, hypertriglyceridemia, and recurrent bone fractures upon osteoporosis. His cognitive development was normal. He was the only child of unrelated parents and he was childless. None of his relatives presented with premature aging features.

The other VUS in the LMNA gene was detected in patient P17 who was addressed for a clinical phenotype evocative of cEDS. This variant was located in the 3'UTR region of Lamin A-encoding transcripts (NM_170707.4: c. $1698+17 \mathrm{G}>\mathrm{A}$ ) and in the last codon of Lamin Cencoding transcripts (NM_005572.3) (c.1715G > A; p.(Arg572His)). Unfortunately, no segregation study could be performed in the patient's first-degree relatives.

\section{Discussion}

We report the outcomes of 4 years of molecular genetic diagnosis by high throughput sequencing on a cohort of 66 patients mostly affected with premature aging syndromes, either nosologically classified or not, using a panel of 82 genes. To the best of our knowledge, this panel is the only available in France and in Europe to offer a wide molecular genetics exploration of disorders including features of premature aging. Other panels, mostly involved in the molecular diagnosis of connective tissue disorders or more restrained nosologic entities among premature aging syndromes, partially share genes with the panel we present.

This panel allowed us to provide molecular genetics results for patients from all over France and, in a few cases, from French overseas territories or abroad. As expected, and as seen in Fig. 1, more than half of the patients' samples received in Marseille La Timone Molecular Genetics Laboratory were issued from the southern part of France. This is due to the strong previous interactions established in other diagnostic contexts with other southern French University Hospitals, and their facilitation by the establishment of financial agreements among regional hospitals in France. Nonetheless, the other cases were addressed from the rest of French territories or abroad, probably due to the unique availability of a large molecular screening in this 
kind of rare syndromes where, in some cases, large clinical overlaps can be observed. Additionally, this may be explained by the long-lasting involvement of our medical team in translational research and diagnosis of premature aging disorders, namely linked to Lamins $\mathrm{A} / \mathrm{C}[1,10,13$, 27, 57-62].

While for the global cohort of patients $(n=66)$ the diagnostic yield (i.e. identification of pathogenic or likely pathogenic variants) was of about $1 / 4(26 \%)$, by further dividing the cohort in two groups, depending on the strength of the clinical suspicion towards a nosologically defined progeroid syndrome vs. an unspecified progeroid disorder (UPS), we observed that the diagnostic yield largely relied on this parameter, with almost $40 \%$ resolved cases in the first category of patients. When those groups were further subdivided in three ("specified clinical suspicion other than connective tissue disorders", "connective tissue disorders" and "unspecified progeroid disorders"), the diagnostic yields were respectively 62, 26 and $10 \%$. Indeed, this allows to observe, as expected, that the lowest yield is obtained in UPS, but a low yield is also retrieved in connective tissue disorders. This group of patients represents about $1 / 3$ rd of the cases addressed to our laboratory, with a clinical diagnosis or suspicion of EDS (Fig. 3 and data not shown). The International EDS Consortium recently defined 13 EDS subtypes and listed 19 causal genes [28]. In our panel, we analyze more than $50 \%$ of them, including the most frequently mutated ones (11/19). In France, to date, there is no available diagnostic panel exploring the 19 genes. For example, the extended panel of Tübingen in Germany, studies $90 \%$ of these 19 genes (17/19). Our panel thus represents the most extensive offer in France for EDS molecular diagnosis to date but it will be further implemented to include 19/19 genes in a future version. On the other hand, the number of patients that we received for this clinical indication points to a probably uncovered diagnostic need in France, together with the fact that it's often hard to have a precise clinical suspicion given the high phenotypic variability of the EDS subtypes as well as the clinical overlap among EDS subtypes and other connective tissue disorders, making it interesting to use a large panel including most of the genes involved in those disorders with features of premature aging. This was also the reason why, although no specific gene has been identified yet, patients with a clinical diagnosis of hypermobile EDS were accepted and included in the molecular analysis. Indeed, more widely, many syndromes that are associated with the 82 genes included in our panel have overlapping clinical features $[28,63]$. We report in this work 20 pathogenic or likely pathogenic variants in different clinical indications, 9 of them being novel. Among these novel variants 4 were non-sense and 2 frameshifting. We detected novel variants and in some cases reclassified them as pathogenic or likely pathogenic according to ACMG criteria, relative to genetic databases, literature and patients' clinical history (Table 1): for RECQL4 c.2263C > T; p.(Arg755Trp) and c.2415 2419dup; p.(Arg807fs113Ter) variants in patient P2 presenting with clinical RTS, for GORAB c.546A $>$ T; p.(Glu182Asp) and c.859C > T; p.(Arg287Ter) in patient P5 presenting with clinical CL. Indeed, even if most pathogenic variants of GORAB (alias: SCYL1BP1) responsible of GO are nonsense, homozygous missense variants in compound heterozygosity with null alleles have already been associated with GO in previous publications $[64,65]$.

We also reclassified the ERCC6 (c.2291 T > C; p.(Leu764Ser)) variant in patient P7 carrying a deletion of ERCC6 on the other allele and a clinical diagnosis of late onset Cockayne syndrome; of note, Ghai et al. described a patient affected with Cokayne syndrome carrying almost the same deletion as P7 associated to a splice variant [66]. For $A L D H 18 A 1$ the homozygous variant: c. $1499 \mathrm{G}>\mathrm{T}$; p.(Gly500Val) was reclassified in patient P13 presenting with features of CL; for COL5A1, the (c.1884_1891del; p.(Asp629Phefs16Ter)) nonsense variant was reclassified in patient P14 presenting with classical EDS. Other novel variants already classified as pathogenic by Intervar, an ACMG variant classification tool, were observed in PRDM5 (homozygous variant: c.1036C > T; p.(Arg346Ter)) in patient P15 presenting with Brittle Cornea syndrome and in WRN (c.2313 T > A; p.(Cys771Ter)) associated with another already described nonsense variant in patient P8 presenting with classical Werner syndrome.

Other previously described pathogenic variants were identified in patients affected with syndromes as diverse as SHORT, Buschke-Ollendorf syndrome, BranchioOculo-Facial Syndrome (BOFS), and Oculodentodigital Dysplasia.

Among the 16 VUS observed, 3 were re-classified as benign by studying their segregation in first degree relatives' DNAs.

Interestingly, three variants were detected in LMNA: one known pathogenic variant and two novel VUS, whose pathogenicity could not be proven for the moment, due to the lack of segregation studies or functional in vitro analyses. One of the LMNA VUS (NM_170707.4: c.1016C > T; p.(Ala339Val)) was found in a male proband (patient P18) who presented with an adult-onset progeroid phenotype suggestive of Atypical Werner Syndrome (AWS), described in patients presenting with some clinical signs of WS and carrying heterozygous missense LMNA variants [20, 22, 67-69]. Furthermore, the WRN gene, whose coding sequence NM_000553.5 was entirely covered by the genomic sequencing, did not show any pathogenic or likely pathogenic variants. We consider the LMNA 
p.(Ala339Val) variant as likely responsible for the patient's phenotype, although it doesn't fulfill ACMG criteria for (likely) pathogenicity [35]. Indeed, Ala339 is a very conserved residue and the LMNA gene has only a few wellknown polymorphic variants (SNPs), making every undescribed variant very suspect of being pathogenic, especially if compatible with previously described laminopathies. Moreover, it is located nearby other previously reported AWS mutations [22, 68, 69].

The other LMNA VUS we observed (NM_005572.3: c.1715G > A; p.(Arg572His)) affected only Lamin Cencoding transcripts, being otherwise located in the 3' UTR region of Lamin A-encoding transcripts, in patient P17, who was addressed for a suspicion of cEDS. It would be very interesting to perform the variant segregation study in this patient's first relatives since, to the best of our knowledge, no variant affecting uniquely Lamin C isoforms has ever been described in human. Indeed, most laminopathies, including atypical progeria syndromes [24, 26, 70-72], have been associated to missense mutations affecting both Lamin A and C isoforms, and the mutation of only Lamin $\mathrm{C}$ isoforms may be compatible with a relatively mild clinical phenotype.

The familial segregation of these VUS and eventual functional analyses will be important to determine whether or not they are major pathogenic factors at the origin of the clinical phenotypes of the patients or only possible phenotype modifiers.

Patient P11 carrying the heterozygous c.1003C > T; p.(Arg335Trp) pathogenic variant in LMNA (NM 170707.4), already described in [48-54], presented since a young age with a severe progeroid phenotype of acroosteolysis and cutaneous premature aging associated with heart rhythm disturbances; by giving us the authorization of publishing her pictures, the patient allows the medical community to become more familiar with the spectrum of clinical phenotypes (ranging from isolated dilated cardiomyopathy to premature aging phenotypes) linked to this particular $L M N A$ mutation, as already reported for other LMNA mutations.

Finally, the identification of pathogenic variants allowed us to offer genetic counselling and propose prenatal testing: four prenatal tests were performed in the three families with mutations in GORAB, RECQL4 and TFAP2A.

\section{Conclusion}

Globally, the NGS molecular exploration of 66 patients using a panel of 82 genes associated with premature aging syndromes allowed us to confirm or establish a final diagnosis in $26 \%$ (about $1 / 4$ th) of the cases, while segregation analysis of first-degree relatives would help to reclassify the VUS identified in about $15 \%$ of the cohort.

The identification of a molecular diagnosis for these rare but often severe disorders, allowed to provide genetic counseling to the families and to propose prenatal diagnosis, contributing to personalized genomic, medical healthcare.

In order to further improve our panel's diagnostic yield, clinicians will need to be sensitized to the utility of completing the segregation analyses in the families with VUS and sending at once the trios DNAs (affected patient and parents) whenever possible; also, research projects including functional in vitro analysis on human biological samples may be designed in order to include patients with VUS if the segregation analysis is compatible with a likely pathogenic effect, in order to provide arguments for it and refine the molecular diagnosis. On the other hand, for patients for whom only ACMG classes 1 or 2 were observed (which represent overall, about $60 \%$ of the patients of our cohort) further studies in a research context may be proposed.

Alternatively, the French government has financed the first two National high throughput whole genome sequencing platforms for trios; indeed, analysis of both parents and child are well known to improve the diagnostic performance [73, 74]. These WGS platforms have been established in the context of the "Medicine France Genomics 2025” Plan (https://solidarites-sante.gouv.fr/ systeme-de-sante-et-medico-social/recherche-etinnovation/france-genomique), aiming to sustain and improve molecular genetic diagnosis of patients affected with rare disorders and therapeutic follow up of patients affected with cancer in the context of genomic, personalized medicine, allowing to further integrate scientific advances into healthcare and to facilitate the access to innovation to all patients. These patients and their relatives may also be candidate for this second level screening in the next years.

\section{Additional file}

Additional file 1: Tables S1. Alphabetical list of genes included in the NGS panel "premature ageing syndromes and laminopathies". AD: automal dominant; AR: autosomal recessive; Mi: mitochondrial; XLR: X linked recessive; XLD: $X$ linked dominant; Smu: somatic mutation; IC: isolated cases. (XLSX $33 \mathrm{~kb}$ )

\section{Abbreviations}

aCGH: array comparative genomic hybridization; ACMG: American College of Medical Genetics; APS: Atypical progeroid syndromes; AWS: Atypical Werner Syndrome; BOFS: Branchio-Oculo-Facial Syndrome; BS: Bloom syndrome; cEDS: Classical EDS; CL: Cutis Laxa; ClEDS: Classical-Like EDS; CRB-

TAC: Biological Resources Center, Tissues-DNA-Cells; DCM: Dilated Cardiomyopathy; DNA: Desoxyribonucleic acid; EDS: Ehlers Danlos Syndrome; HGPS: Hutchinson Gilford Progeria Syndrome; MAD-B: Type B mandibuloacral dysplasia; RD: Restrictive Dermopathy; RTS: Rothmund Thomson Syndrome; SHORT: Short stature, Hyperextensibility of joints, Ocular depression, Rieger anomaly, Teething delay; UPS: Unspecified Progeroid Disorder; VUS: Variant of Unknown Significance; WS: Werner syndrome 


\section{Acknowledgements}

We thank all the patients and their families for their precious collaboration to this work.

We also thank the AP-HM Data Protection Representative for helping us in guaranteeing patients' data protection, complying with the RGPD regulation.

\section{Authors' contributions}

MG performed the retrospective study of the whole cohort, organized the results, prepared a critical analysis of them and drafted the paper; ADSG is the medical practictioner in charge for the molecular genetics diagnostic tests in the laboratory, she supervised the work as well as the article organization, critical discussion and writing. VB is the engineer that works on the panel, SS, NP,FG, KK, GM, SG, JS, CP, JL, LA, PC, GC,VL, AM, CC, LM, PE, and NL sent their patients' samples to the molecular genetics laboratory for genetic testing and participated to the critical review of the manuscript. All authors read and approved the final manuscript.

\section{Funding}

The authors received no personal funding for this work. Publication charges were supported thanks to the attribution to our research team (Aging Prenylation and Cancer, Inserm U1251-Marseille Medical Genetics, AixMarseille Université) of the "Prix Départemental pour la Recherche en Provence 2017" by the Conseil Départemental des Bouches du Rhône.

\section{Availability of data and materials}

The excel tables contained in the main text and/or in the supporting materials section will be freely downloadable. Please contact the corresponding author for other data requests.

\section{Ethics approval and consent to participate}

This study fully complies with the European RGPD directive ( $\left.n^{\circ} 2016 / 679\right)$ : the use of genetic and clinical data issued from molecular genetics diagnostics procedures were validated from the Data Protection Officer of APHM (Assistance Publique des Hôpitaux de Marseille), in agreement with the European and French regulation.

\section{Consent for publication}

All the patients or the parents/legal guardians for minors, provided written informed consent for genetic analysis following the French regulation (L1131-1-1 Code de la Santé Publique). Specific consents for publication were additionally obtained from the patients or the parents / legal guardians of the minor patients whose clinical presentation is further developed in the article, namely including their pictures.

\section{Competing interests}

The authors declare that they have no competing interests.

\footnotetext{
Author details

${ }^{1}$ Department of Medical Genetics, Assistance Publique Hopitaux de Marseille, Marseille, France. ${ }^{2}$ Aix Marseille Univ, INSERM, MMG, Marseille, France. ${ }^{3}$ Medical Genetics Unit 2, L'Archet Hospital, Nice, France. ${ }^{4}$ Hospices Civils de Lyon, Genetic Department and National HHT Reference Center, Femme-Mère-Enfants Hospital, F-69677 Bron, France. ${ }^{5}$ Université Claude Bernard Lyon 1, F-69100 Villeurbanne, France. ${ }^{6}$ Genetics Department, AP-HP, Robert-Debré University Hospital, Paris, France. ${ }^{7}$ Department of Medical Genetics, CHU Toulouse, Purpan Hospital, 31059 Toulouse, France. ${ }^{8}$ Department of Genetics, Reims University Hospital, Reims, France. ${ }^{9}$ Department of Genetics, Centre Hospitalier de Chambéry- Hôtel-dieu, Chambery, France. ${ }^{10}$ Internal Medicine, CHU Toulouse, Rangueil Hospital, Toulouse 3 University Hospital Center, Toulouse, France. ${ }^{11}$ Internal Medecine, CHR Metz-Thionville, Thionville, France. ${ }^{12}$ Department of Genetics, Le Havre Hospital, F76600 Le Havre, France. ${ }^{13}$ Department of Genetics, CHU de Caen, Avenue de la Cote de Nacre, 14000 Caen, France. ${ }^{14}$ Department of Clinical Genetics, Lille University Hospital, CHU, Lille, France. ${ }^{15}$ CRB-TAC (Biological Ressource Center-Tissues, DNA, Cells), Assistance Publique Hopitaux de Marseille, Marseille, France.
}

Received: 17 May 2019 Accepted: 30 August 2019

Published online: 11 December 2019

\section{References}

1. Cau P, Navarro C, Harhouri K, Roll P, Sigaudy S, Kaspi E, et al. Nuclear matrix, nuclear envelope and premature aging syndromes in a translational research perspective. Semin Cell Dev Biol. 2014 May;29:125-47.

2. Larsen NB, Hickson ID. RecQ Helicases: Conserved Guardians of Genomic Integrity. Adv Exp Med Biol. 2013;767:161-84.

3. Fu W, Ligabue A, Rogers KJ, Akey JM, Monnat RJ. Human RECQ helicase pathogenic variants, population variation and "missing" diseases: HUMAN MUTATION. Hum Mutat. 2017;38(2):193-203.

4. Croteau DL, Popuri V, Opresko PL, Bohr VA. Human RecQ helicases in DNA repair, recombination, and replication. Annu Rev Biochem. 2014;83(1):519-52.

5. Yu C-E, Oshima J, Fu Y-H, Wijsman EM, Hisama F, Alisch R, et al. Positional cloning of the Werner's syndrome gene. Science. 1996;272(5259):258-62.

6. Ellis NA, Groden J, Ye T-Z, Straughen J, Lennon DJ, Ciocci S, et al. The Bloom's syndrome gene product is homologous to RecQ helicases. Cell. 1995:83(4):655-66.

7. Kitao S, Shimamoto A, Goto M, Miller RW, Smithson WA, Lindor NM, et al. Mutations in RECQL4 cause a subset of cases of Rothmund-Thomson syndrome. Nat Genet. 1999;22(1):82-4.

8. Wilson BT, Stark Z, Sutton RE, Danda S, Ekbote AV, Elsayed SM, et al. The Cockayne syndrome natural history (CoSyNH) study: clinical findings in 102 individuals and recommendations for care. Genet Med. 2016;18(5):483.

9. Calmels N, Botta E, Jia N, Fawcett H, Nardo T, Nakazawa Y, et al. Functional and clinical relevance of novel mutations in a large cohort of patients with Cockayne syndrome. J Med Genet. 2018;55(5):329-43.

10. De Sandre-Giovannoli A, Bernard R, Cau P, Navarro C, Amiel J, Boccaccio I, et al. Lamin a truncation in Hutchinson-Gilford progeria. Science. 2003;300(5628):2055.

11. Eriksson M, Brown WT, Gordon LB, Glynn MW, Singer J, Scott L, et al. Recurrent de novo point mutations in Lamin a cause Hutchinson-Gilford progeria syndrome. Nature. 2003;423(6937):293-8.

12. Goldman RD, Shumaker DK, Erdos MR, Eriksson M, Goldman AE, Gordon LB, et al. Accumulation of mutant Lamin a causes progressive changes in nuclear architecture in Hutchinson-Gilford progeria syndrome. Proc Natl Acad Sci U S A. 2004;101(24):8963-8.

13. Barthélémy F, Navarro C, Fayek R, Da Silva N, Roll P, Sigaudy S, et al. Truncated prelamin a expression in HGPS-like patients: a transcriptional study. Eur J Hum Genet. 2015 Aug;23(8):1051-61.

14. Agarwal AK, Fryns J-P, Auchus RJ, Garg A. Zinc metalloproteinase, ZMPSTE24, is mutated in mandibuloacral dysplasia. Hum Mol Genet. 2003; 12(16):1995-2001.

15. Navarro CL, De Sandre-Giovannoli A, Bernard R, Boccaccio I, Boyer A, Genevieve D, et al. Lamin a and ZMPSTE24 (FACE-1) defects cause nuclear disorganization and identify restrictive dermopathy as a lethal neonatal laminopathy. Hum Mol Genet. 2004;13(20):2493-503.

16. Navarro CL, Cadiñanos J, De Sandre-Giovannoli A, Bernard R, Courrier S, Boccaccio I, et al. Loss of ZMPSTE24 (FACE-1) causes autosomal recessive restrictive dermopathy and accumulation of Lamin a precursors. Hum Mol Genet. 2005 Jun 1;14(11):1503-13.

17. Navarro CL, Poitelon Y, Lévy N. Lamines A et syndromes progéroïdesUne farnésylation persistante aux conséquences dramatiques. Méd Sci. 2008;24(10):833-40

18. Worman HJ, Ostlund C, Wang Y. Diseases of the nuclear envelope. Cold Spring Harb Perspect Biol. 2010;2(2):a000760.

19. Navarro CL, Esteves-Vieira V, Courrier S, Boyer A, Duong Nguyen T, Huong LTT, et al. New ZMPSTE24 (FACE1) mutations in patients affected with restrictive dermopathy or related progeroid syndromes and mutation update. Eur J Hum Genet. 2014;22(8):1002-11.

20. Chen L, Lee L, Kudlow BA, Dos Santos HG, Sletvold O, Shafeghati Y, et al. LMNA mutations in atypical Werner's syndrome. Lancet. 2003;362(9382):440-5.

21. Madej-Pilarczyk A, Kmieć T, Fidziańska A, Rekawek J, Niebrój-Dobosz I, Turska-Kmieć A, et al. Progeria caused by a rare LMNA mutation p.S143F associated with mild myopathy and atrial fibrillation. Eur J Paediatr Neurol Off J Eur Paediatr Neurol Soc. 2008 Sep;12(5):427-30.

22. Renard D, Fourcade G, Milhaud D, Bessis D, Esteves-Vieira V, Boyer A, et al. Novel LMNA Mutation in atypical werner syndrome presenting with ischemic disease. Stroke. 2009:40(2) Available from: https://www.ahajournals. org/doi/10.1161/STROKEAHA.108.531780. Cited 10 Mar 2019. 
23. Madej-Pilarczyk A, Rosińska-Borkowska D, Rekawek J, Marchel M, Szaluś E, Jabłońska $S$, et al. Progeroid syndrome with scleroderma-like skin changes associated with homozygous R435C LMNA mutation. Am J Med Genet A. 2009;149A(11):2387-92.

24. Garg A, Subramanyam L, Agarwal AK, Simha V, Levine B, D'Apice MR, et al. Atypical Progeroid syndrome due to heterozygous missense LMNA mutations. J Clin Endocrinol Metab. 2009:94(12):4971-83.

25. Liang L, Zhang H, Gu X. Homozygous LMNA mutation R527C in atypical Hutchinson-Gilford progeria syndrome: evidence for autosomal recessive inheritance. Acta Paediatr Oslo Nor 1992. 2009;98(8):1365-8.

26. Doubaj Y, De Sandre-Giovannoli A, Vera E-V, Navarro CL, Elalaoui SC, Tajir M, et al. An inherited LMNA gene mutation in atypical progeria syndrome. Am J Med Genet A. 2012;158A(11):2881-7.

27. Soria-Valles C, Carrero D, Gabau E, Velasco G, Quesada V, Bárcena C, et al. Novel LMNA mutations cause an aggressive atypical neonatal progeria without progerin accumulation. J Med Genet. 2016;53(11):776-85.

28. Malfait F, Francomano C, Byers P, Belmont J, Berglund B, Black J, et al. The 2017 international classification of the Ehlers-Danlos syndromes. Am J Med Genet C Semin Med Genet. 2017;175(1):8-26.

29. Mohamed M, Voet M, Gardeitchik T, Morava E. Cutis Laxa. In: Halper J, editor. Progress in heritable soft connective tissue diseases. Dordrecht: Springer Netherlands; 2014. p. 161-84. Available from: http://link.springer. com/10.1007/978-94-007-7893-1_11. Cited 2 Feb 2019.

30. Desvignes J-P, Bartoli M, Delague V, Krahn M, Miltgen M, Béroud C, et al. VarAFT: a variant annotation and filtration system for human next generation sequencing data. Nucleic Acids Res. 2018;46:W545-53.

31. Robinson JT, Thorvaldsdóttir H, Winckler W, Guttman M, Lander ES, Getz G, et al. Integrative genomics viewer. Nat Biotechnol. 2011;29(1):24.

32. Desmet F-O, Hamroun D, Lalande M, Collod-Béroud G, Claustres M, Béroud C. Human splicing finder: an online bioinformatics tool to predict splicing signals. Nucleic Acids Res. 2009;37(9):e67.

33. Schwarz JM, Rödelsperger C, Schuelke M, Seelow D. MutationTaster evaluates disease-causing potential of sequence alterations. Nat Methods. 2010;7(8):575.

34. Salgado D, Desvignes J-P, Rai G, Blanchard A, Miltgen M, Pinard A, et al. UMDpredictor: a high-throughput sequencing compliant system for pathogenicity prediction of any human CDNA substitution. Hum Mutat. 2016;37(5):439-46.

35. Richards S, Aziz N, Bale S, Bick D, Das S, Gastier-Foster J, et al. Standards and guidelines for the interpretation of sequence variants: a joint consensus recommendation of the American College of Medical Genetics and Genomics and the Association for Molecular Pathology. Genet Med Off J Am Coll Med Genet. 2015;17(5):405-24.

36. Pujol LA, Erickson RP, Heidenreich RA, Cunniff C. Variable presentation of Rothmund-Thomson syndrome. Am J Med Genet. 2000;95(3):204-7.

37. Wang LL, Gannavarapu A, Kozinetz CA, Levy ML, Lewis RA, Chintagumpala MM, et al. Association between osteosarcoma and deleterious mutations in the RECQL4 gene in Rothmund-Thomson syndrome. J Natl Cancer Inst. 2003 May 7;95(9):669-74

38. Siitonen HA, Sotkasiira J, Biervliet M, Benmansour A, Capri Y, CormierDaire $V$, et al. The mutation spectrum in RECQL4 diseases. Eur J Hum Genet. 2009;17(2):151-8

39. Wiest T, Herrmann O, Stögbauer F, Grasshoff U, Enders H, Koch MJ, et al. Clinical and genetic variability of oculodentodigital dysplasia. Clin Genet. 2006;70(1):71-2.

40. Hellemans J, Debeer P, Wright M, Janecke A, Kjaer KW, Verdonk PC, et al Germline LEMD3 mutations are rare in sporadic patients with isolated melorheostosis. Hum Mutat. 2006;27(3):290.

41. Reiber J, Sznajer Y, Posteguillo EG, Müller D, Lyonnet S, Baumann C, et al. Additional clinical and molecular analyses of TFAP2A in patients with the branchio-oculo-facial syndrome. Am J Med Genet A. 2010; 152A(4):994-9.

42. Dyment DA, Smith AC, Alcantara D, Schwartzentruber JA, Basel-Vanagaite L, Curry CJ, et al. Mutations in PIK3R1 cause SHORT syndrome. Am J Hum Genet. 2013:93(1):158-66

43. Reversade B, Escande-Beillard N, Dimopoulou A, Fischer B, Chng SC, Li Y, et al. Mutations in PYCR1 cause cutis laxa with progeroid features. Nat Genet. 2009;41(9):1016-21.

44. Kretz R, Bozorgmehr B, Kariminejad MH, Rohrbach M, Hausser I, Baumer A, et al. Defect in proline synthesis: pyrroline-5-carboxylate reductase 1 deficiency leads to a complex clinical phenotype with collagen and elastin abnormalities. J Inherit Metab Dis. 2011;34(3):731-9.

45. Fischer-Zirnsak B, Escande-Beillard N, Ganesh J, Tan YX, Al Bughaili M, Lin $A E$, et al. Recurrent De novo mutations affecting residue Arg138 of
Pyrroline-5-carboxylate synthase cause a Progeroid form of autosomaldominant cutis Laxa. Am J Hum Genet. 2015;97(3):483-92.

46. Wenstrup RJ, Florer JB, Willing MC, Giunta C, Steinmann B, Young F, et al. COL5A1 haploinsufficiency is a common molecular mechanism underlying the classical form of EDS. Am J Hum Genet. 2000;66(6):1766-76.

47. Al-Hussain H, Zeisberger SM, Huber PR, Giunta C, Steinmann B. Brittle cornea syndrome and its delineation from the kyphoscoliotic type of Ehlers-Danlos syndrome (EDS VI): report on 23 patients and review of the literature. Am J Med Genet A. 2004;124(1):28-34

48. Lakdawala NK, Funke BH, Baxter S, Cirino AL, Roberts AE, Judge DP, et al. Genetic testing for dilated cardiomyopathy in clinical practice. J Card Fail. 2012;18(4):296-303.

49. Bollati M, Barbiroli A, Favalli V, Arbustini E, Charron P, Bolognesi M. Structures of the Lamin a/C R335W and E347K mutants: implications for dilated cardiolaminopathies. Biochem Biophys Res Commun. 2012;418(2):217-21.

50. Stallmeyer B, Koopmann M, Schulze-Bahr E. Identification of novel mutations in LMNA associated with familial forms of dilated cardiomyopathy. Genet Test Mol Biomarkers. 2012;16(6):543-9.

51. Carboni N, Politano L, Floris M, Mateddu A, Solla E, Olla S, et al. Overlapping syndromes in laminopathies: a meta-analysis of the reported literature. Acta Myol Myopathies Cardiomyopathies Off J Mediterr Soc Myol. 2013;32(1):7-17.

52. Pugh TJ, Kelly MA, Gowrisankar S, Hynes E, Seidman MA, Baxter SM, et al. The landscape of genetic variation in dilated cardiomyopathy as surveyed by clinical DNA sequencing. Genet Med. 2014;16(8):601.

53. Zaragoza MV, Hakim SA, Hoang V, Elliott AM. Heart-hand syndrome IV: a second family with LMNA -related cardiomyopathy and brachydactyly: letter to the editor. Clin Genet. 2017;91(3):499-500.

54. Lambert J-C, Baudart P, De Sandre-Giovannoli A, Molin A, Marcelli C. Lamin a/C gene (LMNA) mutation associated with laminopathy: a rare cause of idiopathic acro-osteolysis. Joint Bone Spine. 2019;86(4):525-7.

55. Giunta C, Steinmann B. Compound heterozygosity for a disease-causing G1489E [corrected] and disease-modifying G530S substitution in COL5A1 of a patient with the classical type of Ehlers-Danlos syndrome: an explanation of intrafamilial variability? Am J Med Genet. 2000;90(1):72-9.

56. Malfait F, De Paepe A. Molecular genetics in classic Ehlers-Danlos syndrome. Am J Med Genet C: Semin Med Genet. 2005;139C(1):17-23.

57. Lo Cicero A, Saidani M, Allouche J, Egesipe AL, Hoch L, Bruge C, et al. Pathological modelling of pigmentation disorders associated with Hutchinson-Gilford progeria syndrome (HGPS) revealed an impaired melanogenesis pathway in iPS-derived melanocytes. Sci Rep. 2018;8(1):9112.

58. Harhouri K, Frankel D, Bartoli C, Roll P, De Sandre-Giovannoli A, Lévy N. An overview of treatment strategies for Hutchinson-Gilford progeria syndrome. Nucl Austin Tex. 2018:9(1):246-57.

59. Harhouri K, Navarro C, Depetris D, Mattei M-G, Nissan X, Cau P, et al. MG132-induced progerin clearance is mediated by autophagy activation and splicing regulation. EMBO Mol Med. 2017;9(9):1294-313.

60. Elouej S, Beleza-Meireles A, Caswell R, Colclough K, Ellard S, Desvignes JP, et al. Exome sequencing reveals a de novo POLD1 mutation causing phenotypic variability in mandibular hypoplasia, deafness, progeroid features, and lipodystrophy syndrome (MDPL). Metabolism. 2017;71:213-25.

61. Harhouri K, Navarro C, Baquerre C, Da Silva N, Bartoli C, Casey F, et al. Antisense-based Progerin downregulation in HGPS-like patients' cells. Cells. 2016:5(3):31.

62. Ambrosi P, Kreitmann B, Lepidi H, Habib G, Levy N, Philip N, et al. A novel overlapping phenotype characterized by lipodystrophy, mandibular dysplasia, and dilated cardiomyopathy associated with a new mutation in the LMNA gene. Int J Cardiol. 2016;209:317-8.

63. Morava E, Guillard M, Lefeber DJ, Wevers RA. Autosomal recessive cutis laxa syndrome revisited. Eur J Hum Genet. 2009;17(9):1099-110.

64. Hennies HC, Kornak U, Zhang H, Egerer J, Zhang X, Seifert W, et al. Gerodermia osteodysplastica is caused by mutations in SCYL1BP1, a Rab-6 interacting golgin. Nat Genet. 2008;40(12):1410-2.

65. Al-Dosari M, Alkuraya FS. A novel missense mutation in SCYL1BP1 produces geroderma osteodysplastica phenotype indistinguishable from that caused by nullimorphic mutations. Am J Med Genet A. 2009;149A(10):2093-8.

66. Ghai SJ, Shago M, Shroff M, Yoon G. Cockayne syndrome caused by paternally inherited $5 \mathrm{Mb}$ deletion of $10 \mathrm{q} 11.2$ and a frameshift mutation of ERCC6. Eur J Med Genet. 2011;54(3):272-6.

67. Doh YJ, Kim HK, Jung ED, Choi SH, Kim JG, Kim BW, et al. Novel LMNA gene mutation in a patient with atypical Werner's syndrome. Korean J Intern Med. 2009;24(1):68-72. 
68. Motegi S, Yokoyama Y, Uchiyama A, Ogino S, Takeuchi Y, Yamada K, et al. First Japanese case of atypical progeroid syndrome/atypical Werner syndrome with heterozygous LMNA mutation. J Dermatol. 2014:41(12):1047-52.

69. Yanhua X, Suxian Z. Cerebral Haemorrhage in a Young patient with atypical Werner syndrome due to mutations in LMNA. Front Endocrinol. 2018;9:433.

70. Csoka AB, Cao H, Sammak PJ, Constantinescu D, Schatten GP, Hegele RA. Novel Lamin a/C gene (LMNA) mutations in atypical progeroid syndromes. J Med Genet. 2004:41(4):304-8.

71. Brown CA, Lanning RW, McKinney KQ, Salvino AR, Cherniske E, Crowe CA, et al. Novel and recurrent mutations in Lamin a/C in patients with EmeryDreifuss muscular dystrophy. Am J Med Genet. 2001;102(4):359-67.

72. van der Kooi AJ, Bonne G, Eymard B, Duboc D, Talim B, Van der Valk M, et al. Lamin a/C mutations with lipodystrophy, cardiac abnormalities, and muscular dystrophy. Neurology. 2002;59(4):620-3.

73. Wright CF, Fitzgerald TW, Jones WD, Clayton S, McRae JF, van Kogelenberg M, et al. Genetic diagnosis of developmental disorders in the DDD study: a scalable analysis of genome-wide research data. Lancet. 2015;385(9975):1305-14.

74. Tammimies K, Marshall CR, Walker S, Kaur G, Thiruvahindrapuram B, Lionel AC, et al. Molecular diagnostic yield of chromosomal microarray analysis and whole-exome sequencing in children with autism spectrum disorder. JAMA. 2015;314(9):895-903.

\section{Publisher's Note}

Springer Nature remains neutral with regard to jurisdictional claims in published maps and institutional affiliations.

Ready to submit your research? Choose BMC and benefit from:

- fast, convenient online submission

- thorough peer review by experienced researchers in your field

- rapid publication on acceptance

- support for research data, including large and complex data types

- gold Open Access which fosters wider collaboration and increased citations

- maximum visibility for your research: over $100 \mathrm{M}$ website views per year

At $\mathrm{BMC}$, research is always in progress.

Learn more biomedcentral.com/submissions 